\title{
DEVELOP A GIS-BASED CONTEXT-AWARE SENSOR NETWORK DEPLOYMENT ALGORITHM TO OPTIMIZE SENSOR COVERAGE IN AN URBAN AREA
}

\author{
Meysam Argany ${ }^{1, *}$, Mir-Abolfazl Mostafavi ${ }^{2}$ \\ ${ }^{I}$ Department of Remote Sensing \& GIS, Faculty of Geography, University of Tehran, Tehran, Iran - argany@ut.ac.ir \\ ${ }^{2}$ Department of Geomatics, Faculty of Forestry, Geography, and Geomatics, Laval University, Quebec, Canada - \\ mir-abolfazl.mostafavi@scg.ulaval.ca
}

Technical Commission I

KEYWORDS: sensor network, deployment, optimization, context-aware, urban area

\begin{abstract}
:
Adequate coverage is an important issue in geosensor networks in order to fulfill the sensing applications in urban areas. GIS as well as Optimization methods are widely used to distribute geosensors in the network to achieve the desired level of coverage. Most of the algorithms applied in urban domain suffer from the lack of considering real environmental information. In this paper, the problem of placing sensors to get optimum coverage is studied by investigating the concept of urban contextual information in sensor network. Then, a local GIS-based context-aware framework of sensor network deployment optimization method is introduced. Obtained results of our algorithm under different working conditions and applications show the effectiveness of our approach.
\end{abstract}

\section{INTRODUCTION}

In recent years, sensor networks have been increasingly used for different applications in smart cities ranging from urban environmental monitoring, tracking of moving objects, development of smart cities and smart transportation system, etc. (Nittel 2009). A sensor network usually consists of numerous wireless devices deployed in a region of interest inside the cities as well as other locations (Lewis 2004). Despite the advances in the sensor network technology, the efficiency of a sensor network for collection and communication of the information may be constrained by the limitations of geosensors deployed in the network nodes. These restrictions may include sensing range, battery power, connection ability, memory, and limited computation capabilities (Ghosh and Das 2008). These limitations create challenging problems for the users of the sensor networks, which has pushed researchers from different disciplines in recent years to study various problems related to the design and deployments of efficient sensor networks in urban areas (Ghosh 2004; Thai et al. 2008). Also sensor networks have some limitations when it comes to the modeling, monitoring, and detecting urban environmental processes (Thai et al. 2008; Li, $\mathrm{Li}$, and Vasilakos 2013). Urban environmental elements like virtual and real obstacles, which exist in both static and dynamic natures, are also important to be considered in a realistic sensor networks deployment in urban areas. These restrictions usually affect the network coverage of the sensors. Spatial coverage of sensor networks has different definition according to different applications (Aziz, Aziz, and Ismail 2009; Ahmed, Kanhere, and Jha 2005; Ghosh and Das 2008; Huang and Tseng 2005; Adriaens, Megerian, and Potkonjak 2006; Akbarzadeh et al.
2013; Argany et al. 2011; Paul and Sato 2017). Other examples of such elements include contextual information of the geosensors environment and physical urban phenomena in the network (Sun and Sauvola, 2003; Park, Savvides, and Srivastava 2000; Abu-Mahfouz and Hancke 2018). It is necessary to know how to use such information to make an appropriate and efficient sensor network deployment. For this purpose, one needs to introduce relevant models of the phenomena type, the accessibility or inaccessibility of the observation area, urban environmental conditions, spatial relations between sensors as well as urban deployment area, and related information availability. The complexity of urban area, as the sensing environment of sensor networks, with the presence of diverse obstacles may result in several uncovered areas in the sensing field (Ghosh 2004). Consequently, sensor placement affects how well a region of a city is covered by geosensors as well as the cost for constructing the network and create its relations (Thai et al. 2008; Shit, Sharma, and Puthal 2018; Priva and Sivakumar 2018). Hence, a fundamental issue in a sensor network deployed in an urban area is the optimization of its spatial coverage. Several optimization algorithms have been developed and applied in recent years to meet this criterion. Most of these algorithms often rely on oversimplified sensors, network models, and city model representations ( $\mathrm{Li}, \mathrm{Li}$, and Vasilakos 2013). In addition, they do not consider environmental information such as city terrain models, man-made infrastructures, human and living creatures, and the presence of diverse urban obstacles in optimization process.

The impact of the quality of initial datasets used to deploy geosensors in the networks is another aspect of the complexity of wireless sensor network in urban areas (Aziz, Aziz, and Ismail

* Corresponding author 
2009). Therefore, choosing the way of deploying sensors and the data accuracy needed to set up a sensor network in an optimal manner are difficult due to the abundance of available deployment algorithms as well as design of a consistent, reliable,

\section{METHODS}

In order to develop a context aware method for sensor network deployment we need to precise the implication of the context and context awareness in this work. In order to provide a meaningful definition for the concept of "context" for sensor network deployment, the concept of sensor shall take the role of the main object of interest in such a definition. Here, the main object of interest is a sensor. Sensor network is considered as the object environment, which includes information on the sensors, for example, sensor position, orientation, and its spatial relations with other sensors in the network. Physical environment is composed of spatial objects in a given urban area in which the sensor is placed. It also may refer to the spatial relations among the objects, the specific locations in the urban environment such as desirable areas to be covered or restricted positions that are forbidden to set up the network. Here, context may also include information on sensor network preferences, objectives, and interests. Therefore, a comprehensive definition of context in sensor networks domain is proposed as follows: "Context is the whole situation, background, or environment of a sensor network. It includes information on sensor itself, the network, and the physical environment and their interactions in a given time."

That being said, for a context-aware sensor network deployment we need to identify different contextual information (CI). Knowledge on $\mathrm{CI}$ for sensor results in awareness of any sensor on its position, orientation, and relation and interaction with other sensors and the physical environment and helps to hence decide on intelligent actions for sensor network optimization.

The contextual information may be very divers in their nature and require different strategies to be categorized in terms of integration in optimization algorithms. CI in sensor network deployment could be classified into spatial, temporal, and thematic information.

Spatial contextual information refers to the ability of defining objects positions, and geometric relations. Spatial CI is not only about $2 \mathrm{D}$ or $3 \mathrm{D}$ position of sensors. A comprehensive framework of spatial contextual information may include sensors orientation, movement, routing, targeting, topology, and spatial dependencies and interactions. Hence, all information of spatial relations, interactions, proximity, and adjacency lie in this category.

Temporal contextual information concerns the temporal information, and the temporal dependencies in data. Temporal information characterizes the dependency of a situation in the sensor network framework with the time, and also indicates an instant or period during which some other CI is known or relevant. The objects and activities in the physical environment may change. For instance position or attributes of an obstacle (e. g. its height) may change during a given period of time. A specific example of temporal CI is the information of a sensor movement and its trajectory in the network. Previous actions and movements of a sensor node may provide useful information for the next actions of current sensor or its neighbors.

Thematic contextual information in sensor networks constitutes the sensor specifications, network objectives, environment and robust network. Thus, study of wireless sensor networks is a challenging task, as it requires multi-disciplinary knowledge and expertise.

specifics, legal rules, etc. The information regarding the nodes names and roles, and their activity in the network is included in this category. Sensors activities may include measurement of the temperature, humidity, sound, or light. In terms of deployment, the type of sensor movement and its trajectory could be the sensor activity inside the network. Node name should be unique in the network in order to make it possible to be recognized and devolve its roles in multi tasks networks. Sensor characteristics are sensor specifications, which have been designed during their manufacturing, e.g., their power supply, battery life, sensing range, temperature resistance, dimensions, input and output terminals, processing power, data storage capacity, send and receive information protocols, and etc. Network objective express the mission of sensor network to be fulfilled. This objective could be various in multi task networks. It may be varied from covering a whole, or a part of study area to monitor a phenomenon, or sensing different characteristics of the environment. Legal rules define specified terms and conditions for constructing and deploying the sensor networks, e.g., in which locations sensor deployment is allowed, or which parameters are permissible to be measured.

Based on mentioned issues on sensor networks deployment in urban areas, this paper presents a GIS-based approach to improve sensor deployment processes by integrating urban geospatial information and knowledge with optimization algorithms. To achieve this objective, the following approach that contains three specific parts is defined. First, a conceptual framework is proposed to show how geosensors and urban contextual information are integrated with a sensor network deployment processes. Then, a local GIS-based context-aware optimization algorithm is developed based on the proposed framework. The extended approach is a generic local algorithm for sensor deployment, which accepts spatial, temporal, and thematic contextual information related to sensors and their distributing locations in different situations. Next, the accuracy assessment and error propagation analysis is conducted to determine the impact of the accuracy of contextual information on the proposed sensor network optimization method.

\subsection{A Conceptual GIS-based Context-Aware Framework for Sensor Network Deployment}

Many parameters directly affect the sensing coverage, for example, topological relations among the sensors in the network, the interactions between the sensors and the environmental elements, and the relationship among the environmental elements themselves. Here, such information and relations are called network Contextual Information (CI). Specifically, CI defines the spatial dependencies between spatially adjacent nodes, nodes and urban obstacles, and obstacles themselves as well as the temporal dependencies between historical movements of nodes in the deployment process. Urban furniture, buildings, poles, etc. can be considered as the urban obstacles. The term of temporal information of sensor movements means the history of previous moves and trajectories of sensors which have had impact on the network coverage as well as their impact on the new probable moves. The so-called CI is used in the proposed 
framework to find good candidates positions of sensor nodes to fill uncovered areas, and decide about the sensor's adequate actions in order to guide sensor network deployment.

The proposed conceptual context-aware framework consists of the following steps. First the appropriate CI is extracted from the city area which is aimed to be studied. After introducing the CI to the framework, spatial and network databases are created. Spatial database contains CI related to the physical urban environment, while the network database comprises the CI belongs to sensors' configuration and relations. Accordingly, a knowledge base is defined considering both databases. In the next step, a reasoning engine is applied using the predefined knowledgebase. The optimization algorithm is also specified regarding the introduced local CI and tasks at hand. Afterward, the rules extracted from the reasoning engine along with the determined optimization method are applied to perform contextaware deployment actions. This may include a sensor move, delete or insert. These actions may change the topology of the network, the configuration of the adjacent nodes, and consequently, the local coverage. As a result, local CI may be updated. Then, the information in the knowledge base is changed and so on. This is an iterative algorithm and these actions are done until the desired level of deployment is achieved. The process of local optimization in the framework means that network configuration is changed locally at each step until the best coverage is obtained by considering the spatial, temporal, and thematic contextual information in the network (Figure 1).

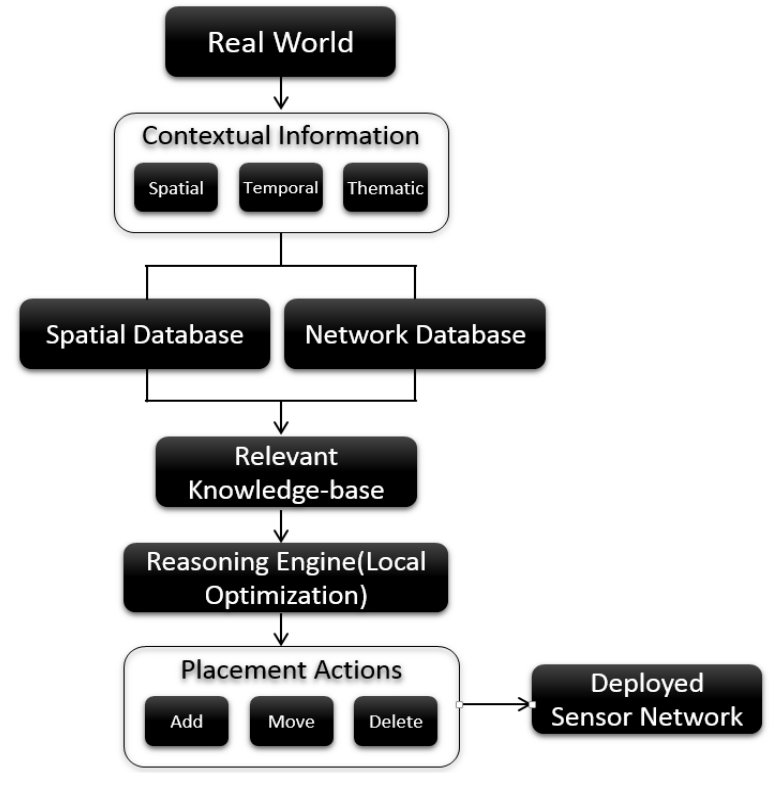

Figure 1: Context-aware sensor network deployment framework

\subsection{Local Context-Aware Optimization Algorithm for Sensor Network Deployment}

According to the proposed framework for sensor network deployment, a local optimization algorithm is developed to tackle the sensors placement problem and maximize the spatial coverage of the network in an urban region. In the proposed algorithm, a set of sensors $\left(S=\left\{s_{1}, \ldots, s_{n}\right\}\right)$ are randomly deployed at the first step. Then, sensors starts to moves based on provided CI. There are some strategies for sensor movements to optimise the network coverage (Karimipour, Argany, and Mostafavi 2014). Next, the coverage $\left(s_{i}\right)$ for each sensor is calculated. There are different definition of coverage in literature. In this study, the blanket coverage is assumed. Blanket coverage requires placing a minimum number of nodes in an environment, such that every point of interest in the sensing area shall be adequately covered regarding tasks at hand (Fan and Jin 2010). Afterward, sensors are sorted in a priority queue, based on their coverage gain $\left(g_{i}\right)$ obtained by considering different $\mathrm{CI}$, and following related moves in the network.

$$
\begin{gathered}
C_{i}=\text { coverage }\left(\operatorname{move}\left(s_{i}, S\right)\right), \forall s_{i} \in S \\
g_{i}=\operatorname{coverage}\left(\operatorname{move}\left(s_{i}, S\right)\right)-\operatorname{coverage}\left(s_{i}, S\right), \forall s_{i} \in S
\end{gathered}
$$

Then the sensor with the maximum gain is selected, which is the sensor that obtained the highest coverage improvement by its movement in the network, and stands at the top of the queue. The movement types of sensors are related to the local CI as well as sensor network mission. By changing the position of the topmost sensor of the queue $\left(s_{u}^{\prime}\right)$, the network configuration is updated.

$$
s_{u}^{\prime}=\operatorname{move}\left(s_{u}, S\right)
$$

Next, the coverage gain of the adjacent sensors of moved sensor is recalculated and their ordering in the priority queue is updated. In the next iteration, the (new) topmost sensor of the queue is chosen to move, and so on. This optimization process is conducted iteratively until one of the predefined stopping criteria is reached (Figure 2). The iteration will be stopped when top most sensor movement do not improve overall coverage of the network. The maximum gain will be defined based on the deployment mission.

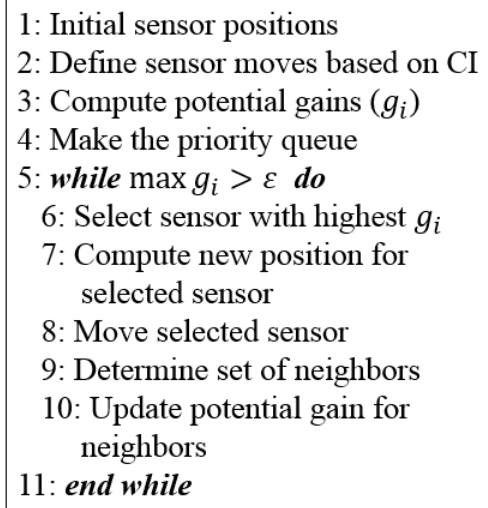

Figure 2: The pseudo-code of the local context-aware algorithm

\subsection{Impact of the Spatial Data Quality on Sensor Network Deployment}

The sensor placement optimization algorithms that are applied in our experimentation use urban spatial information to calculate spatial coverage. This way, visible and invisible objects are identified and hence, covered and uncovered areas in the urban region of interest are defined. The quality of spatial data has a direct impact on the estimation of these values. Among different data quality elements, positional accuracy and completeness were selected to study because of their direct impact on the 
estimation of the visibility (Aziz, Aziz, and Ismail 2009). The positional accuracy may be presented as a small displacement in the position of the objects, which can be either horizontal or vertical or both. Even a few centimeters inaccuracy in horizontal or vertical positions of objects or sensors can block the line-ofsight between a sensor and a target. Same reasoning may be applied for incompleteness of databases.

\section{EXPERIMENTATIONS AND RESULTS}

\subsection{Study Area and Datasets}

The study area of the experiments is a part of the campus of Université Laval, located in Quebec City, with a dimension of $300 \mathrm{~m}$ by $300 \mathrm{~m}$ (Figure 3 ). For the experiments, 12 sensors have been assumed to be deploying as surveillance cameras with 360 degrees horizontal, \pm 90 degrees vertical sensing angle, and 50 meters of effective sensing range.

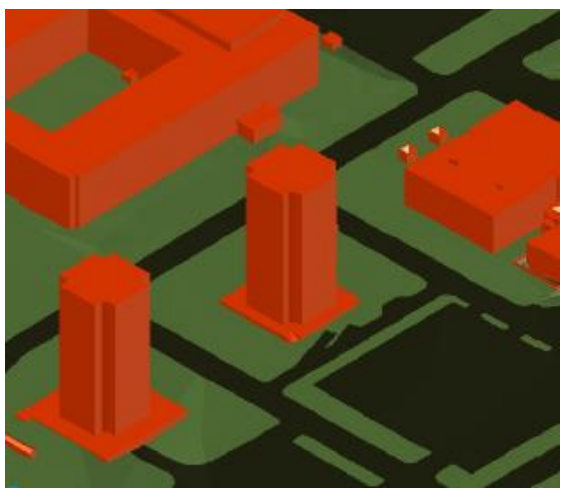

Figure 3: Part of the campus of Université Laval

In order to deploy sensors using the proposed context-aware algorithm, unlike many previous optimization algorithms which do not consider the environment topography or real obstacles, the sensors were deployed over the terrain model of study area. The buildings and other urban features and obstacles have been added over the terrain model containing their horizontal shapes and coordinates as well as their heights (Figure 3). The test site contains the buildings of Library, Faculty of Educational Sciences, Faculty of Philosophy, two main streets, and a parking lot.

\subsection{Different Contextual Information Situations}

3.2.1. Terrain Surface and City Features Model: The first category of $\mathrm{CI}$ is the city surface model of the sensor network distributing area, besides the information of the network. Having this information the height of city objects, and the terrain elevation model in study area is provided, and as a result the obstacles which bared the sensing field of the sensors are introduced. Accordingly, after running the context-aware algorithm, the sensors start to move and the network configuration is changed based on provided CI. In each iteration, sensors find their best positions, over the buildings or on the terrain surface, based on the coverage improvement they might provide.

Figure 4 depicts the coverage improvement during running the optimization algorithm for one of runs which returned the best coverage over 32 runs. It shows that in each iteration, the overall network coverage is improved, while the optimum coverage in this network is reached at just 10 iterations, which is significantly less than other deployment optimization methods with the same initial parameters. For instance, the deployment progress were compared to the CMA-ES (Akbarzadeh et al. 2013) optimization algorithm. In order to avoid the impact of initial positions over the final results (Argany et al. 2012), 32 runs for each method with 32 different initial random positions was performed. The results are presented in Table 1.

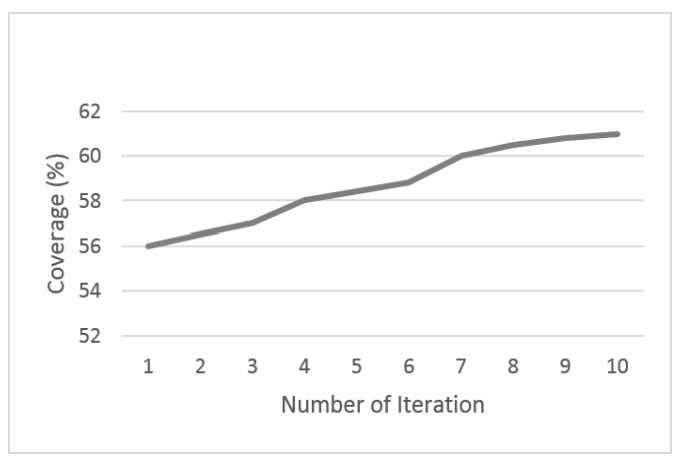

Figure 4: Coverage improvement over iterations for run number 18 which returned best results for the context-aware method

\begin{tabular}{|l|l|l|}
\hline Method & $\begin{array}{l}\text { Avg. Coverage } \\
(\%)\end{array}$ & Best Coverage (\%) \\
\hline Context-Aware & 64.14 & 66.26 \\
\hline CMA-ES & 61.76 & 64.44 \\
\hline
\end{tabular}

Table 1: Results of Context-Aware algorithm compared to CMA-ES optimization algorithm

3.2.2. Urban Legal Restrictions: Urban legal restrictions are considered as the next category of CI which was introduced to the sensor network optimization context-aware algorithm. In this study, inside the streets and parking areas (dark green area in Figure 3), and top of two towers (red towers in Figure 3) were assumed to be unauthorized places for sensor installation. Considering restricted areas in context-aware optimization, sensor action is changed, and new moves are defined within the optimization process. Again, 32 runs with different random initial positions were applied to avoid unexpected impact of initial sensor positions. Table 2 shows the result of 5 best runs.

\begin{tabular}{|ccc|}
\hline Run No. & $\begin{array}{c}\text { Overall } \\
\text { Coverage (\%) }\end{array}$ & $\begin{array}{c}\text { Num. of } \\
\text { Iterations }\end{array}$ \\
\hline 21 & 61.78 & 8 \\
26 & 61.59 & 7 \\
20 & 60.87 & 9 \\
14 & 59.16 & 8 \\
5 & 58.42 & 7 \\
\hline
\end{tabular}

Table 2: Results of 5 best runs for Context-Aware algorithm considering urban restrictions as $\mathrm{CI}$ 
3.2.3. Desirability of coverage: Desirability of coverage is another type of urban thematic CI which is introduced to be considered in the proposed context-aware optimization process. Suppose that there are some places in the campus, where sensors should not be installed, but there is a high interest on those regions to be covered. In this study, "Avenue des Sciences Humaines", the street between towers and the library building was introduced as the area with high interest to be covered, while it is unauthorized zone for sensors to be deployed. Table 3 presents results for both coverage of interested zone plus overall coverage for the best run of algorithm as well as the average result over 32 runs.

\begin{tabular}{|l|l|l|}
\hline & $\begin{array}{l}\text { Avg. } \\
\text { Coverage } \\
(\%)\end{array}$ & $\begin{array}{l}\text { Best } \\
\text { Coverage } \\
(\%)\end{array}$ \\
\hline $\begin{array}{l}\text { Coverage on } \\
\text { "Avenue des }\end{array}$ & 85.07 & 86.55 \\
$\begin{array}{l}\text { Sciences } \\
\text { Humaines" }\end{array}$ & 63.51 & 65.83 \\
\hline $\begin{array}{l}\text { Overall } \\
\text { Coverage }\end{array}$ & & \\
\hline
\end{tabular}

Table 3: Results of Context-Aware algorithm using desirability of coverage in an urban region

3.2.4. Evaluation the Minimum Number of Sensors: To evaluate the investigation of minimum number of sensors needed in each sensor group network to cover a region of interest in an urban area, we studied the sensor deployment over the campus using four groups of $8,12,16$, and 20 sensors in the network. The results shows that approximately 50 percent of sensors in is applied to cover the region of interest, when the number of sensors are 8 and 12. While, in the group of 16 and 20, there is still 6 sensors which is used to cover the "Avenue des Sciences Humaines". This means that the targeted street is covered using maximum of 6 sensors, and the average coverage $85.07 \%$ reaches by adding just 2 sensors from the previous test, which had 4 sensors to be deployed. In next tests, by considering 16 and 20 sensors, the overall coverage over the whole study area is improved, while the coverage on "Avenue des Sciences Humaines" remain unchanged due to reach the optimum number of sensors to cover this area. This confirms the capability of proposed method to optimize not only the overall and interested coverage of the network but also the number of sensors used to perform desired actions. Table 4 has the result of this investigation.

\begin{tabular}{|c|c|c|c|c|}
\hline & $\begin{array}{c}\text { Total } \\
\text { Num. of } \\
\text { Sensors }\end{array}$ & $\begin{array}{l}\text { Num. of } \\
\text { Sensors } \\
\text { to Cover } \\
\text { RoI }\end{array}$ & $\begin{array}{c}\text { Avg. } \\
\text { Coverage } \\
(\%)\end{array}$ & $\begin{array}{c}\text { Best } \\
\text { Cover } \\
\text { age } \\
(\%)\end{array}$ \\
\hline $\begin{array}{lr}\text { Coverage } & \text { on } \\
\text { "Avenue } & \text { des } \\
\text { Sciences Humaines" }\end{array}$ & \multirow[t]{2}{*}{8} & \multirow[t]{2}{*}{ 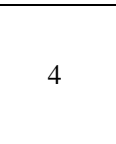 } & 69.36 & 71.10 \\
\hline Overall Coverage & & & 51.67 & 53.22 \\
\hline $\begin{array}{lr}\text { Coverage } & \text { on } \\
\text { "Avenue des } & \text { des } \\
\text { Sciences Humaines" }\end{array}$ & \multirow[t]{2}{*}{12} & \multirow[t]{2}{*}{6} & 85.07 & 86.55 \\
\hline Overall Coverage & & & 63.51 & 65.83 \\
\hline $\begin{array}{lr}\text { Coverage } & \text { on } \\
\text { "Avenue } & \text { des } \\
\text { Sciences Humaines" }\end{array}$ & 16 & 6 & 85.07 & 86.55 \\
\hline
\end{tabular}

\begin{tabular}{|l|l|l|c|c|}
\hline Overall Coverage & & & 65.29 & 68.04 \\
\hline $\begin{array}{l}\text { Coverage on } \\
\text { "Avenue des } \\
\text { Sciences Humaines" }\end{array}$ & \multirow{2}{*}{20} & \multirow{6}{*}{6} & 85.07 & 86.55 \\
\hline Overall Coverage & & & & \\
\cline { 1 - 3 } & & & 66.86 & 69.62 \\
\hline
\end{tabular}

Table 4: Results of increasing number of sensors to cover the region of interest

Finally, to investigate the impact of positional accuracy and completeness of the dataset on the spatial coverage of a sensor network, we prepared 5 maps with different resolutions for the campus area, which contain the terrain surface information plus the height of urban features like buildings and other obstacles. The resolution variation is from $500 \mathrm{~cm}$ (low resolution) to 50 $\mathrm{cm}$ (high resolution) and a map with $10 \mathrm{~cm}$ resolution is considered as the ground truth dataset to validate the results. All maps are from the same area, which previous tests were done. The experimentation consists of deploying eight sensors considering the first category of CI (terrain and urban surface model) inside the study area. Similar to previous tests, the proposed context-aware optimization algorithm was run 32 times over the study area in order to reduce the probable impact of the initial sensor position on final results. Then, for each map, average coverage, and best coverage of 32 runs were calculated. Table 5 shows the results. The results show that both average and best coverage are improved by increasing the data resolution. The proposed algorithm performs better in higher resolution because the urban features and obstacles are appeared with more details. In return, when the optimum sensor positions have been found from different testing resolutions are applied on the $10 \mathrm{~cm}$ reference map, the average and best coverage are reduced. This could be interpreted with the same reason. When the contextaware algorithm find optimum sensor positions using a lower resolution data, then we applied same positions over a higher resolution map, we considered more details of a higher resolution map to calculate coverage using positions that are obtained based on lower data resolutions.

\begin{tabular}{|l|l|l|l|l|}
\hline $\begin{array}{l}\text { Reso } \\
\text { lutio } \\
\mathrm{n} \\
(\mathrm{cm})\end{array}$ & $\begin{array}{l}\text { Avg. } \\
\text { coverage } \\
(\%)\end{array}$ & $\begin{array}{l}\text { Best } \\
\text { coverage } \\
(\%)\end{array}$ & $\begin{array}{l}\text { Best coverage } \\
\text { from best } \\
\text { configuration } \\
\text { over 10cm } \\
\text { resolution }(\%)\end{array}$ & $\begin{array}{l}\text { Average } \\
\text { coverage } \\
\text { over } \\
10 \mathrm{~cm} \\
\text { resolutio } \\
\text { n(\%) }\end{array}$ \\
\hline 500 & 48.61 & 51.36 & 48.39 & 45.92 \\
\hline 300 & 48.85 & 51.73 & 47.42 & 46.28 \\
\hline 200 & 50.39 & 52.96 & 51.60 & 50.05 \\
\hline 100 & 51.67 & 53.22 & 51.68 & 49.85 \\
\hline 50 & 53.85 & 55.59 & 53.20 & 51.69 \\
\hline
\end{tabular}

Table 5: Results obtained from the context-aware method, considering the urban surface model.

\section{CONCLUSIONS}

The purpose of this paper was certainly not to overcomplicate the optimization process, but rather to find a flexible methodology that can locally accommodate all relevant urban information that would have an impact on sensor placement. To do so, a local optimization framework was introduced. The extended 
optimization algorithm can come up with different sensor placement configuration according to the various circumstances, environmental information, and/or sensor parameters encountered. Consequently, if there are any changes in sensor parameters or urban environment, the context-aware algorithm can simply take in new contextual inputs and regenerate a new sensor placement design adapted to the new situation. The outstanding advantage of the proposed context-aware algorithm was that it was designed independent of any specific CI. Thus, it is able to take into consideration different types of information based on specific network applications, urban requirements, and tasks at hand.

\section{REFERENCES}

Abu-Mahfouz, A. M. and Hancke, G. P., 2018. Localised information fusion techniques for location discovery in wireless sensor networks. Journal of Sensor Networks, inderscienceonline.com

Adriaens, Jacob, Seapahn Megerian, and Miodrag Potkonjak. 2006. Optimal Worst-Case Coverage of Directional Field-ofView Sensor Networks. 3rd Annual IEEE Communications Society on Sensor and Ad Hoc Communications and Networks. Ieee, 336-45. doi:10.1109/SAHCN.2006.288438.

Ahmed, Nadeem, SS Kanhere, and S Jha. 2005. The Holes Problem in Wireless Sensor Networks: A Survey. ACM SIGMOBILE Mobile Computing and Communication Review 1 (2): 1-14. http://dl.acm.org/citation.cfm?id=1072992.

Akbarzadeh, Vahab, Christian Gagne, Marc Parizeau, Meysam Argany, and Mir Abolfazl Mostafavi. 2013. Probabilistic Sensing Model for Sensor Placement Optimization Based on Line-of-Sight Coverage. IEEE Transactions on Instrumentation and Measurement 62 (2): 293-303.

doi:10.1109/TIM.2012.2214952.

Argany, Meysam, Mir Abolfazl Mostafavi, Vahab Akbarzadeh, Christian Gagne, and Reda Yaagoubi. 2012. I MPACT OF THE QUALITY OF SPATIAL 3D CITY MODELS ON SENSOR NETWORKS. Geomatica 66 (4): 291-305.

Argany, Meysam, Mir Abolfazl Mostafavi, Farid Karimipour, and Christian Gagné. 2011. A GIS Based Wireless Sensor Network Coverage Estimation and Optimization: A Voronoi Approach. Transaction on Computational Science XIV 6970: 151-72.

http://www.springerlink.com/index/K17K3H824G48L06N.pdf.

Aziz, NAA, KA Aziz, and WZW Ismail. 2009. Coverage Strategies for Wireless Sensor Networks. World Academy of Science, Engineering and Technology 50: 145-50. http://www.waset.ac.nz/journals/waset/v26/v26-24.pdf.

Fan, Gaojun, and Shiyao Jin. 2010. Coverage Problem in Wireless Sensor Network: A Survey. Journal of Networks 5 (9): 1033-40. doi:10.4304/jnw.5.9.1033-1040.

Ghosh, Amitabha. 2004. Estimating Coverage Holes and Enhancing Coverage in Mixed Sensor Networks. In 29th Annual IEEE International Conference on Local Computer Networks, 68-76. IEEE (Comput. Soc.).

\section{doi:10.1109/LCN.2004.53.}

Ghosh, Amitabha, and Sajal K. Das. 2008. Coverage and Connectivity Issues in Wireless Sensor Networks: A Survey. Pervasive and Mobile Computing 4 (3): 303-34. doi:10.1016/j.pmcj.2008.02.001.

Huang, CF, and YC Tseng. 2005. A Survey of Solutions to the Coverage Problems in Wireless Sensor Networks. Journal of Internet Technology 1: 1-9.

http://people.cs.nctu.edu.tw/ yctseng/papers.pub/sensor04jit.doc.

Karimipour, Farid, Meysam Argany, and Mir Abolfazl Mostafavi. 2014. Spatial Coverage Estimation and Optimization in Geosensor Networks Deployment. In Wireless Sensor Networks, From Theory to Applications, edited by M M Ibrahiem and S. Ramakrishnan, 59-83. CRC Press, Taylor \& Francis Group.

https://books.google.com/books?hl=en\&lr=\&id=KUTSBQAA QBAJ\&oi=fnd $\& p g=P P 1 \&$ ots $=$ mqio7TBQ4n\&sig $=185 \mathrm{ZkwxH} 1$ 2 tGf-jUSutAgqGyhOI\#v=onepage \&q\&f=false.

Lewis, F L. 2004. Wireless Sensor Networks. Edited by C. S. Raghavendra, Krishna M. Sivalingam, and Taieb Znati. Smart Environments: Technologies, Protocols, and Applications. Boston: Kluwer Academic Publishers, 1-18. doi:10.1007/b117506.

Li, By Mo, Zhenjiang Li, and Athanasios V Vasilakos. 2013. A Survey on Topology Control in Wireless Sensor Networks : Taxonomy , Comparative Study , and Open Issues. Proceedings of the IEEE, 101 (12): 2538-2557.

Nittel, Silvia. 2009. A Survey of Geosensor Networks: Advances in Dynamic Environmental Monitoring. Sensors (Basel, Switzerland) 9 (7): 5664-78. doi:10.3390/s90705664.

Park, Sung, A Savvides, and MB Srivastava. 2000. SensorSim: A Simulation Framework for Sensor Networks. In Proceedings of the 3rd ACM International Workshop on Modeling, Analysis and Simulation of Wireless and Mobile Systems, 104-11. New York, NY, USA. doi:10.1145/346855.346870.

Paul, A. K., Sato, T., 2017. Localization in Wireless Sensor Networks: A Survey on Algorithms, Measurement Techniques, Applications and Challenges. Journal of Sensor and Actuator Networks, 6(4) 24, mdpi.com

Priya, C.B.; Sivakumar, S. 2018. A survey on localization techniques in wireless sensor networks. International Journal of Engineering and Technology, 7, 1-3.

Sun, J.-Z., and J. Sauvola. 2003. Towards a Conceptual Model for Context-Aware Adaptive Services. Proceedings of the 8th International Scientific and Practical Conference of Students, Post-Graduates and Young Scientists. Modern Technique and Technologies. MTT'2002 (Cat. No.02EX550). Ieee, 90-94. doi:10.1109/PDCAT.2003.1236265.

Shit, R.C., Sharma, S., Puthal, D., 2018. Location of Things (LoT): A Review and Taxonomy of Sensors Localization in IoT 
Infrastructure. IEEE Communications Surveys \& Tutorials, ieexplore.ieee.org, doi:10.1109/COMST.2018.2798591

Thai, My T., Feng Wang, David Hongwei Du, and Xiaohua Jia. 2008. Coverage Problems in Wireless Sensor Networks:

Designs and Analysis. International Journal of Sensor

Networks 3 (3): 191-203. doi:10.1504/IJSNET.2008.018482. 